\title{
El desarrollo rural y la gestión del territorio
}

\section{Rural development and territorial management}

\author{
Javier Delgadillo Macías \\ Felipe Torres Torres
}

Investigadores del Instituto de Investigaciones Económicas de la Universidad Autónoma de México Miembros del Sistema Nacional de Investigadores javierdelma@gmail.com felipet@seridor.unam.mx 


\section{RESUMEN}

Se reconoce al medio rural como el entorno territorial donde se producen los alimentos y las materias primas que la sociedad consume, y el lugar donde vive la gente que realiza estas actividades. En un sentido más amplio, también se ve al medio rural como la suma de elementos geográficos que agrupan componentes naturales como recursos bióticos, geológicos, climatológicos y ambientales a los que se atribuyen valores fundamentales para el desarrollo sustentable de las comunidades y de la sociedad en general. Sin embargo, la realidad de lo que sucede al interior del mundo rural se presenta en la actualidad de manera más compleja y rebasa con mucho estos aspectos, ya que la visión de lo rural se sitúa en una dimensión más amplia que la de sólo considerar sus espacios como productores de bienes materiales o como generadores de beneficios económicos en el mercado. Aún es más complicada esta realidad rural al observar la presencia de factores críticos de índole productivo, social, cultural, ambiental e institucional, que se presentan de manera recurrente y generalizados afectando los territorios y comunidades rurales del país.

Palabras clave: Gestión territorial, desarrollo rural, políticas públicas, planeación regional, participación local.

Clasificación JEL: O18, Q18, R58, R51

\section{ABSTRACT}

It acknowledges the rural milieu as the territorial environment where food and the raw materials that society consumes are produced, and as the place where the people that perform these activities live. In a broader sense, the rural milieu is also considered as the sum of geographical elements that concentrate natural components such as biotic, geological, climatic and environmental resources, to which we abscribe fundamental values for the sustainable development of the communities and society in general. Never the less, what really take place inside the rural world is nowadays more complex and goes for beyond these issues, due to the fact that the rural vision is located in a larger dimension than that of only considering its spaces as producers of material goods or as generators of economic benefits in the market. Said rural reality becomes more complicated still when we identify the presence of critical factors of a productive, social, cultural, environmental and institutional nature, that frequently repeat themselves affecting the rural territories of the country. Accordingly, territorial management is an alternative for the new rural development

Keywords: Territorial management, rural development, public policies, regional planning, local participation.

JEF Clasification: $\mathrm{O}_{18}, \mathrm{Q} 18, \mathrm{R}_{5} 8, \mathrm{R}_{51}$

Revista de Economía, vol. XXVII, núm. 74, primer semestre de 2010. 


\section{Presentación}

Se reconoce al medio rural como el entorno territorial donde se producen los alimentos y las materias primas que la sociedad consume, y el lugar donde vive la gente que realiza estas actividades. En un sentido más amplio, también se ve al medio rural como la suma de elementos geográficos que agrupan componentes naturales como recursos bióticos, geológicos, climatológicos y ambientales a los que se atribuyen valores fundamentales para el desarrollo sustentable de las comunidades y de la sociedad en general.

Sin embargo, la realidad de lo que sucede al interior del mundo rural se presenta en la actualidad de manera más compleja y rebasa con mucho estos aspectos, ya que la visión de lo rural se sitúa en una dimensión más amplia que la de sólo considerar sus espacios como productores de bienes materiales o como generadores de beneficios económicos en el mercado. Aún más complicada esta realidad rural al observarse la presencia de factores críticos de índole productivo, social, cultural, ambiental e institucional, que se presentan de manera recurrente y generalizados a lo largo y ancho del territorio nacional.

Ante estas situaciones, enfoques como el territorial-rural (Echeverri, 2009) o el agro-territorial (como lo considera Moyano, 2008) reconocen la importancia de la multidimensionalidad (recursos, factores, procesos, actores e instituciones) como un criterio básico para comprender los nuevos procesos y como el instrumento de análisis para la operación de políticas públicas a favor de una mejor organización de los espacios rurales. Estos enfoques revaloran el papel de la agricultura como un factor necesario para la generación de empleo y riqueza, y como un importante soporte material para la conservación de los espacios naturales, sin embargo, expresan que la actividad agrícola por si sola es insuficiente para mantener un tejido social vivo y dinámico en muchas zonas rurales.

Es en este punto donde cobra importancia el enfoque territorial del desarrollo rural; un enfoque que considera los territorios rurales como sujetos de las políticas públicas y de una nueva institucionalidad para el desarrollo rural; un enfoque que sustituye la operación de políticas sectoriales por otro basado en la integralidad de factores y en una lógica de planeación orientada al territorio; un enfoque que da relevancia a las implicaciones sociales, culturales y ecológicas presentes en cada unidad territorial. 
Las dimensiones que abarca este enfoque pueden resumirse en los siguientes puntos:

1. El desarrollo rural es parte del desarrollo general de un país, de un estado o de una región.

2. En México, derivado del peso que tiene la pobreza rural y del dualismo entre agricultura empresarial y campesina, el concepto de desarrollo rural está íntimamente asociado a la transformación de la agricultura campesina y la lucha contra la pobreza en el campo, hasta el punto que con frecuencia erróneamente se confunden.

3. Las estrategias unilaterales de modernización agrícola no permiten alcanzar un desarrollo sostenible del medio rural. Por ejemplo, cuando se determinan aumentos considerables de producción en ingresos, tienden a concentrarse en grupos de agricultores y regiones cada vez más reducidos, y a demandar cuantiosas subvenciones que, además de ser inequitativas, generan distorsiones en los mercados regionales y en el ámbito nacional. Como contraposición, la puesta en práctica de políticas de desarrollo rural con lógica territorial, acento en la diversificación multisectorial (distintas aunque complementarias a las tradicionales políticas sectoriales) y destinadas al conjunto de la población rural, abren la posibilidad de constatar en las regiones mexicanas, la entrada a procesos de desarrollo rural incluyentes en lo social e integrados en lo económico y territorial.

4. En el marco de la lógica territorial del desarrollo rural, este enfoque le asigna importancia a la competitividad económica de cada territorio, con el propósito de mejorar la calidad de la vida rural; sin embargo, se insiste en que dicha competitividad no cumple su propósito central si no se concibe su impulso mediante la participación organizada de los actores locales en programas especialmente diseñados para aprovechar los recursos materiales e inmateriales de cada zona o región considerada, si no se promueve la diversificación socioeconómica existente, si no se impulsa la multisectorialidad y, sobre todo, si no se vincula al medio rural con los pueblos, barrios, suburbios y áreas pobladas de las zonas rurales más desarrolladas y de las pequeñas y medianas ciudades. 
5. La diversificación de las actividades productivas, el aprovechamiento de las sinergias entre diversos sectores, y los encadenamientos entre actividades productivas y no productivas que permitirían en el mediano plazo aumentar la competitividad del territorio, deben ir acompañadas de programas y acciones impulsadas por el sector público enfocadas entre otras acciones en mejoras en los sistemas locales de comercialización, fomento de las artesanías y otros productos tradicionales, impulso a las agroindustrias y otras industrias locales, promoción del turismo rural y ecológico y otros servicios.

En el presente artículo se abordan aspectos de política pública para la gestión del desarrollo rural a partir de elementos clave de este nuevo enfoque y se discuten criterios que debe contener la institucionalidad que demanda la gestión del desarrollo social, económico y territorial del país.

\section{Los problemas del desarrollo en el medio rural}

Las funciones tradicionales que se le han conferido al medio rural en el desarrollo son la producción de alimentos, junto con el aprovisionamiento de las materias primas requeridas por otras ramas y procesos productivos que conforman el conjunto de la actividad económica. Ambas actividades son consideradas de bajo valor agregado, por lo cual los términos de intercambio respecto a otros productos en general resultan desfavorables al sector primario. De cualquier manera, en la medida que los procesos productivos del campo se ven expuestos y son altamente dependientes a las oscilaciones de los fenómenos naturales como sequías, heladas, ataques de plagas o inundaciones, este debe ser compensado con otro tipo de asignaciones, principalmente factores financieros y tecnológicos de diverso tipo que operan en forma dominante a través de políticas públicas diseñadas por el Estado. Dichas políticas en su diseño y ejecución, se convierten a la vez en su fuente de legitimación en el medio rural.

Estas compensaciones asumen la forma de subsidios a la producción, a los insumos, a la comercialización, a los precios finales o al conjunto de la cadena; están presentes en todos los países del mundo y de su monto, negociación, formas de operación e incluso gestión, depende el éxito tanto de la producción agrícola como del equilibrio social y territorial en el medio rural. 
Es obvio que la jerarquía del medio rural está relacionada con las distintas visiones que asume el Estado en la conducción de la economía, pero sobre todo con la posibilidad de adoptar o no un nuevo enfoque para su desarrollo. En el caso de México, con el ascenso de una economía protegida, los subsidios al campo adquirieron un carácter corporativo y clientelar, sin diferenciaciones regionales, ni en la heterogeneidad existente entre los productores, o la consideración de la diversidad de los sistemas de producción existentes y que deben tomar en cuenta al menos para definir montos y temporalidad de las asignaciones. Ello permitió mantener relativamente bajos los precios de los alimentos pero sometieron al campo a una grave descapitalización y con el tiempo a una crisis agrícola sin precedentes.

Dentro del modelo vigente las prioridades han sido mantener una política de libre mercado que además de repetir los errores estructurales en la definición de subsidios, no considera diferencias entre las actividades territoriales, económicas, culturales o sociales del medio rural, respecto del resto de los sectores. Sus estrategias macroeconómicas más definidas estriban en el control de la inflación, junto con una mayor racionalidad en el gasto público.

Ello se ha traducido en la eliminación gradual de los subsidios al campo, que se recrudece con los recortes actuales al presupuesto de egresos, pero además se encuentra combinado con un asistencialismo clásico, basado en la asignación de un monto directo por superficie sembrada y otras compensaciones indirectas a las familias pobres. Esta directriz no sólo ha profundizado la crisis agrícola y su consecuente dependencia alimentaria con el exterior, sino también evidencia la negación implícita en las políticas neoliberales en lo que se refiere a reconocer que el medio rural ha acrecentado su diversidad de funciones y ambientes territoriales, aunque también se reconoce la necesidad de impulsar a su interior procesos de modernización teniendo en cuenta otras palancas de desarrollo que las mismas dinámicas globales han generado y que están definidas por un alto componente territorial del desarrollo.

Este marco problemático debe reconocer también, en un enfoque multisectorial, la diversidad de ambientes rurales que se presentan actualmente. Entre otros, los generados por la expansión de los principales núcleos urbanos del país que han propiciado un nuevo tipo de ruralidad, 
sea por la complementariedad de ingresos con distinto tipo de ocupación, o el alejamiento gradual de actividades rurales tradicionales, o bien que son absorbidos por oportunidades diferentes que generan las ciudades.

También están presentes los nuevo usos conferidos por el desarrollo a los territorios rurales que llevan a diferentes mecanismos de gestión y vinculación entre distintos tipos de actores sociales y entre diversos niveles territoriales; o bien aquellos territorios que a pesar de ser rurales han quedado marginados en términos reales de las actividades agrícolas y viven de transferencias otorgadas por los gobiernos a través de programas de ayuda social, junto con el envío de remesas externas al entorno. Aquí mismo cabe la consideración de entornos rurales que presentan un alto grado de deterioro ambiental generado por la aplicación intensiva de paquetes tecnológicos dañinos, junto con el uso irracional del agua y la energía, los cuales deben ser rescatados con nuevas propuestas de gestión rural.

Por otra parte, es evidente que los territorios rurales sufren de una descapitalización de sus recursos humanos más valiosos por la vía de una migración que es ya de tipo estructural, lo que además de la imposibilidad de aprovechar positivamente las ventajas del bono demográfico, impide también respaldar cualquier estrategia de recuperación de la seguridad alimentaria o de incorporar a la nuevas fuerzas de desarrollo en el medio rural.

Si bien es cierto que el fenómeno migratorio obedece a la falta de ingresos en el campo, a los bajos niveles de bienestar, al incremento de la pobreza absoluta o bien a la falta de políticas rurales integrales que permitan mejorar los términos de intercambio, lo cierto es que sus efectos se dejan sentir en una disminución de la población que pueda ocuparse en actividades territoriales rurales sean o no de tipo agrícola, lo que a su vez incide en la falta de competitividad que sólo puede recuperarse incorporando a los actores en una nueva dimensión de gestión del desarrollo.

Frente a esta situación de adversidad estructural del sector rural, el enfoque territorial del desarrollo posibilita dimensionar de otra manera las potencialidades de los espacios rurales y de sus pobladores, y enfrentar de mejor manera la crítica situación que envuelve a este sector. Las nuevas estrategias deben adecuarse a los nuevos contextos del desarrollo que operan en condiciones limitadas y competitivas como parte de las dinámicas de los procesos globales, para de esa manera inducir mecanismos creativos que involucren a los actores hacia nuevas formas de gestión territorial del desarrollo. 
Toda esta problemática sugiere abordar sus causas a partir de la consolidación de un modelo de actuación del Estado que contemple el direccionamiento de sus políticas públicas para el medio rural, en una dimensión más amplia que las estrategias sectoriales vigentes, y ensaye, como hoy lo hacen otros países, un modelo alternativo en el cual los territorios rurales sean considerados como posibles sujetos de aplicación de dichas políticas públicas.

\section{La organización del territorio rural con base en su diversidad}

¿Cómo se organizan los territorios rurales para enfrentar su sobrevivencia? Esta respuesta ha estado dada por la actividad económica convencional antes referida, sin embargo, eso mismo se convierte hoy en camisa de fuerza frente a otras perspectivas de desarrollo. El medio rural está cambiando en cuanto a sus funciones por lo que requiere incorporar formas productivas tomando en cuenta la necesidad de conservar a la población en el territorio, que ello se convierta en garantía de su cuidado y sea además fuente para conformar en el largo plazo un bono ambiental y productivo que sostenga a la economía ante la manifiesta escasez de recursos naturales.

Antes el mundo rural tradicional era capaz de organizar pequeñas centralidades y formas de producción, subyugado a los intereses industriales. Las nuevas condiciones productivas, en mucho establecidas por los mercados globales, ponen al servicio de los promotores del desarrollo rural nuevos instrumentos para revertir los problemas de dependencia y establecer otras formas organizativas de tipo local encaminadas a satisfacer demandas internas y externas con base en la integración de energías y organizaciones y en la sustentabilidad ecológica y cultural del sistema rural (Izquierdo, 2005). Actualmente, el desarrollo de una región no está subordinado exclusivamente a su habilidad para atraer funciones o plantas de grandes empresas trasnacionales, sino que depende de su habilidad para estimular iniciativas locales, generar nuevas empresas y llevar una dinámica de innovación territorial.

A ello debe agregarse el paradigma de la sostenibilidad en la solución de problemas territoriales, en la medida que supone que la complejidad territorial funciona como una unidad, además de que su aportación metodológica 
implica una importante dimensión práctica y ética. Consecuentemente, la sostenibilidad territorial debe ser abordada desde un enfoque multidimensional y sistémico, considerando la capacidad sustentadora del territorio en un contexto de uso múltiple que considere los aspectos bióticos y abióticos, sociales, culturales, así como las modalidades de gestión de los territorios y la gobernabilidad de los mismos (Duncan, 2008).

De acuerdo con estas ideas, Izquierdo (2005) señala que el fortalecimiento de los entornos locales orientados al desarrollo rural empieza por dotar de capacidad política, competitiva y financiera a las instituciones más próximas a las personas. Por ello, un nuevo proyecto de política rural se encuentra ante el reto de transformar sus planteamientos desde el objetivo sectorial y productivista del pasado, a un nuevo objetivo social y comprometido en la valorización del territorio en términos ecológicos y culturales. Dentro de este objetivo, la agricultura debe ser el eje articulador del desarrollo rural con base en dos propósitos: primero, producir alimentos sanos para el consumidor, utilizando técnicas y métodos compatibles con el medio ambiente, que preste servicios comerciales como el agroturismo y que a la vez contribuya a la conservación de la biodiversidad y de las tradiciones rurales; $y$, segundo, que conserve el papel central que le corresponde en el conjunto del desarrollo rural, aportando su contribución económica, ocupacional y de mantenimiento de relaciones de complementariedad con la industria y los servicios.

Por tanto, en esta vertiente estratégica que combina lo sectorial con lo territorial, la política territorial debe perfilarse en torno a las siguientes ideas básicas: a) la recuperación de experiencias de gestión operativa local que surjan de iniciativas locales y de las comunidades rurales, y de los programas operativos de desarrollo y diversificación económica de zonas rurales; b) la aplicación de una sólida reforestación de tierras agrícolas, pensada para reactivar tanto la función ecológica de la masa forestal como la función de reactivación de las economías locales; c) la consolidación de un programa de ayudas agroambientales que sirvan para mejorar los aspectos de compensación de renta, pero además se conviertan en incentivos para el desarrollo profesional, personal y social de las comunidades rurales; d) la diversificación de actividades y su integración en el marco social, cultural y ecológico que caracteriza a cada territorio rural; e) la territorialización de los procesos de desarrollo rural, lo que implica un 
proceso de reflexión y análisis de la realidad local, una planificación estratégica territorial de corto, mediano y largo plazos, acompañada de una sólida política de intervención pública que se plantee como objetivos la consolidación de nuevas centralidades en el medio rural, la superación de los déficits de infraestructura, equipamiento y servicios y la corrección de los desequilibrios entre centro y periferia; y f) la integración de diferentes políticas sectoriales: empleo, servicios sociales, promoción económica, ordenación del territorio, conservación del patrimonio, entre otros, junto con los diversos ámbitos de competencia, dentro de una práctica política basada tanto en la unidad de mando como en la cogestión, el consenso al respeto institucional y la subsidiariedad (Izquierdo, 2005).

Esta propuesta de organización de los territorios rurales, particularmente en países como el nuestro, debe vencer no pocos obstáculos. Los territorios rurales incorporan hoy una creciente economía no agrícola que demanda una proporción importante de mano de obra rural, la cual genera casi la mitad del ingreso de sus pobladores; los efectos son diferenciados y para una proporción importante de la población son inviables estos ingresos para su sobrevivencia; sin embargo, el empleo tradicional y los mecanismos de respuesta social e institucional que antes cobijaban los desajustes en la estructura económica del campo, hoy se convierten en procesos y dinámicas organizacionales y productivas que no acaban de asentarse en las regiones mexicanas debido a la dualidad entre modernidad y tradición (Delgadillo, 2008).

Se reconoce que las aplicaciones sectoriales, descoordinadas y escasamente eficaces de los organismos públicos, constituyen en la actualidad la mayor limitación de la administración para fomentar el desarrollo rural integral y territorializado. La complejidad de las nuevas ideas convierten al aparato público en obsoleto para impulsar políticas de desarrollo rural que deben adaptar sus soluciones en función de la especialidad de cada territorio y de cada realidad rural. Sin embargo, la reforma de los instrumentos de intervención administrativa no debe plantearse desde la creación de nuevos organismos al margen de las instituciones existentes, los representantes políticos, las fuerzas sociales, los planes de desarrollo y los servicios públicos que operan en el territorio, sino más bien desde la generación de un pacto y de un espacio común de confluencia para hacer coincidir de manera ordenada todas las fuerzas, recursos e ideas. 
Esta necesidad de coordinar intervenciones públicas y privadas mediante acciones integradas lleva al diseño de una estrategia que, de acuerdo con Izquierdo (2005), puede estar basada en los puntos siguientes:

En primer lugar, la conformación de una fórmula de organización apropiada entre las variadas opciones que lleven a un organismo de coordinación; en segundo lugar, una unidad de mando ya que la coordinación requiere un cierto poder de dirección que a la vez es un elemento de decisión a través de un consenso social e institucional; en tercer lugar, establecer que la coordinación se expresa y se gestiona a través de un plan que traduce la estrategia general y se convierte en el elemento básico de gestión del proceso; y en cuarto lugar, considerar que la gestión del plan y el mantenimiento de los vínculos de coordinación precisan de un instrumento operativo, una gerencia o agencia de desarrollo que interviene técnicamente en el proceso. ${ }^{1}$

Los ejes de la coordinación (fórmula de organización, unidad de mando, plan y gerencia) requieren a su vez de soportes o marcos generales que tienen como función alimentar, renovar y mantener la tensión y el espíritu de colaboración entre la sociedad local y las instituciones. Estos soportes estriban en el pacto territorial o la declaración por el desarrollo local donde se establece un acuerdo tácito sobre las reglas generales del juego que debe ser suscrito por todas las partes interesadas en el desarrollo colectivo; otro soporte tiene que ver con la formulación de canales de participación y corresponsabilidad de la sociedad local que deben funcionar en permanente retroalimentación ya que sin ello la acción ejecutiva coordinada carece de sentido. Gestionar la participación y fomentar la corresponsabilidad es una tarea compleja, pero es imprescindible para reforzar los vínculos entre las personas, la sociedad, el territorio y las instituciones.

\footnotetext{
${ }^{1}$ Véase el texto de Jaime Izquierdo (2005). Manual para agentes de desarrollo rural. Mundi-Prensa, Madrid.
} 


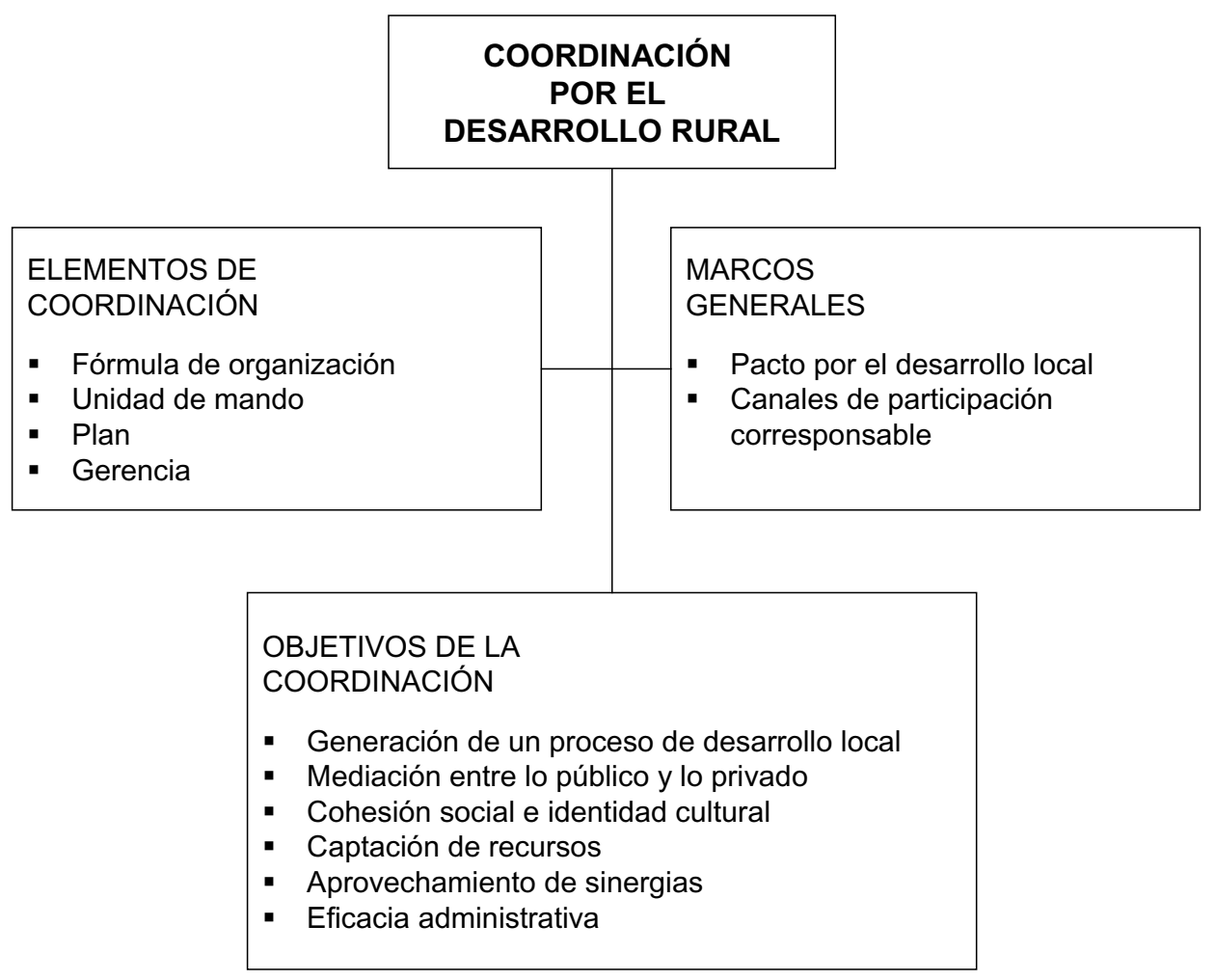

Fuente: Izquierdo, Jaime (2005), op. cit.

\section{La gestión territorial como imperativo del desarrollo rural}

La diversidad de ambientes y contrastes que actualmente presentan los territorios rurales obligan a una reorientación de las políticas públicas, de las estrategias seguidas y de los instrumentos que permitan su reorganización. De manera particular se deben aprovechar las capacidades acumuladas a lo largo del tiempo de los propios actores del desarrollo, entre ellos los funcionarios públicos y los prestadores de servicios profesionales quienes han sido de manera directa corresponsables de gestionar apoyos y procesos en favor del desarrollo local. Pero, junto a ello, es indispensable generar entre los actores rurales nuevas competencias acordes con el contexto actual que les permita estar capacitados para enfrentar la 
crisis del sector, revertir las disparidades sociales y favorecer acciones de participación ciudadana que reditúen en mejores prácticas de desarrollo territorial.

El punto de partida es aceptar, en primer lugar, que el territorio encierra un valor decisivo en el proceso de desarrollo; la identidad territorial y su vinculación con la historia social son elementos primordiales para entender el sistema económico, político y cultural del medio. En segundo lugar, las personas y sus sistemas de organización social, antes instrumentalizadas como mera mano de obra, se revelan hoy como el principal recurso. En tercer lugar, los recursos tangibles o intangibles en el territorio deben ser manejados por personas integradas al sistema territorial para generar procesos de desarrollo local. La articulación entre personas, territorio y recursos debe integrar los conceptos de cooperación y colaboración para generar nuevas formas de gestión territorial que pongan de relieve el cambio cultural en entornos rurales a partir de la síntesis de los elementos tradicionales que conforman la identidad territorial y los recursos procedentes de la innovación urbana y tecnológica; el mundo rural no se conservará si permanece inamovible frente a las nuevas realidades (Izquierdo, 2005).

En el mismo sentido, debe entenderse que el territorio rural no contiene elementos sólo relacionados con la agricultura, sino con el medio ambiente, el paisaje, la salud, el ocio y el bienestar. Los intereses de la población local atribuyen a la agricultura funciones tradicionales que se deben conservar, pero al mismo tiempo se van dando cuenta del beneficio que genera el incorporarle a la agricultura otras funciones distintas a la simple producción de alimentos. Por ello, se manifiesta de acuerdo con apoyar a los agricultores, siempre que las ayudas agrícolas vayan acompañadas del compromiso de respetar al medio ambiente, de utilizar en forma adecuada los recursos naturales, de ahorrar agua, de contribuir a la preservación del paisaje y la conservación del patrimonio cultural; en tal caso, el territorio rural adquiere un carácter multifuncional, el cual se convierte además en un argumento del desarrollo territorial rural. La multifuncionalidad es considerada un atributo de la agricultura y se reconoce a este sector la capacidad para desarrollar múltiples funciones en el ámbito productivo, más allá de su clásica función de producir alimentos, materias primas para la industria textil, productos para la fabricación de fármacos, cultivos agroenergéticos, etcétera. (Moyano, 2008.) 
También la multifuncionalidad es reconocida como un valor organizativo para la planeación territorial rural. De manera particular, cuando se habla de la gestión del desarrollo rural, el enfoque territorial que incluye a la multifuncionalidad ${ }^{2}$ se inscribe dentro de los procesos de desarrollo local, pero de manera organizada y con objetivos integrales enmarcados en distintas dimensiones. La gestión es un diseño del desarrollo y opera a través de actores que encauzan diversas decisiones en el contexto de las potencialidades territoriales rurales. De acuerdo con esto, la gestión se diseña partiendo de la consideración que en el desarrollo local participan un conjunto de elementos individuales y colectivos que tienden a relacionarse entre sí para promover la cohesión interna y la complementariedad de procesos que favorecen la integración territorial. Además de la movilidad ordenada de los recursos locales.

Reforzando este planteamiento, Jaime Izquierdo (2005) hace ver que en el medio rural, el territorio, las personas y los recursos locales requieren desempeñar un protagonismo esencial; asimismo, requiere la renovación de estructuras políticas, sociales, económicas y culturales capacitadas para entender la nueva perspectiva de desarrollo, pero además, permitiendo la interrelación entre infraestructuras, equipamientos, tecnologías, saberes tradicionales, cultura, aptitudes y actitudes. Señala también que a través de la gestión se debe consensuar, relacionar y poner en contacto a las fuerzas sociales, al empresariado y a las instituciones para que el proceso sea integral y aprovechar todas las potencialidades del medio.

Ahora bien, las componentes estratégicas de la gestión territorial incluyen a las instituciones, las asociaciones y las empresas; una primera tarea consiste en reconocer sus identidades territoriales a partir de sus vinculaciones a lo público, lo colectivo y lo privado, toda vez que constituyen la columna mediante la cual se debe organizar al territorio. Por otra parte, la innovación, la formación y la información son básicos para inducir el cambio cultural y conectar a la sociedad local con los nuevos tiempos.

Para poner en marcha políticas de desarrollo rural sustentadas en la gestión local, el entorno institucional debe estructurarse de manera diferente

\footnotetext{
${ }^{2}$ Algunos autores hacen asimilar este concepto de multifuncionalidad con el de multisectorialidad; ambos casos son referentes del concepto de "integralidad" de factores y de procesos.
} 
y adaptarse a las exigencias de los siguientes retos y tendencias. El cuadro siguiente presenta un esbozo de lineamientos estratégicos para la gestión territorial del desarrollo rural:

\begin{tabular}{|l|l|}
\hline $\begin{array}{l}\text { Aplicación de políticas } \\
\text { territoriales }\end{array}$ & $\begin{array}{l}\text { Nuevas políticas públicas que sustituyan } \\
\text { las tradicionales políticas sectoriales. } \\
\text { El objetivo consiste en producir mejores } \\
\text { resulta dos en las dinámicas de desarrollo } \\
\text { rural }\end{array}$ \\
\hline $\begin{array}{l}\text { Animación de la } \\
\text { comunidad para } \\
\text { generar procesos } \\
\text { de participación }\end{array}$ & $\begin{array}{l}\text { El objetivo es avanzar en acciones } \\
\text { de corresponsabilidad de la sociedad } \\
\text { local en el diseño de programas } \\
\text { de desarrollo y en la formulación } \\
\text { de las políticas rurales }\end{array}$ \\
\hline $\begin{array}{l}\text { Incremento de la } \\
\text { eficiencia } \\
\text { institucional }\end{array}$ & $\begin{array}{l}\text { Su propósito consiste en avanzar en } \\
\text { estructuras de gestión adecuadas a cada } \\
\text { programa o iniciativa de desarrollo local }\end{array}$ \\
\hline $\begin{array}{l}\text { Estimulo de la } \\
\text { flexibilidad } \\
\text { institucional }\end{array}$ & $\begin{array}{l}\text { Es un proceso endógeno por medio de } \\
\text { acciones y soluciones que permitan el } \\
\text { funcionamiento complementario y de } \\
\text { cooperación entre administraciones de } \\
\text { distinto ámbito institucional y de } \\
\text { competencias. Los instrumentos de } \\
\text { operación deben estar basados en la } \\
\text { cogestión y la complementariedad } \\
\text { funcional y las competencias } \\
\text { administrativas en lugar de jerarquías } \\
\text { y poder }\end{array}$ \\
\hline
\end{tabular}




\begin{tabular}{|l|l|}
\hline $\begin{array}{l}\text { Mejoramiento y } \\
\text { optimización de la } \\
\text { calidad de las } \\
\text { relaciones de lo público } \\
\text { con lo privado }\end{array}$ & $\begin{array}{l}\text { El principio de corresponsabilidad entre } \\
\text { las administraciones públicas y la sociedad } \\
\text { local debe entenderse como una relación } \\
\text { dinámica y fluida, establecida sobre la base } \\
\text { de la confianza mutua y orientada hacia } \\
\text { el objetivo del desarrollo local. } \\
\text { La descentralización de funciones hasta } \\
\text { ahora exclusivas de la administración } \\
\text { local, la participación en proyectos } \\
\text { empresariales privados, la creación de } \\
\text { sociedades de capital de riesgo, la } \\
\text { liberalización de recursos públicos } \\
\text { ociosos son nuevas opciones y } \\
\text { posibilidades de participación pública } \\
\text { y privada que no han sido exploradas } \\
\text { de manera suficiente. }\end{array}$ \\
\hline
\end{tabular}

\section{Ideas finales}

- A manera de resumen podemos señalar que el enfoque territorial del desarrollo rural es una propuesta centrada en los puntos de interacción entre los sistemas humanos y los sistemas ambientales, a partir de la cual se impulsa la integración de los sistemas productivos rurales y se hace posible generar mejores condiciones de vida e inclusión de grupos sociales relegados.

- Los territorios rurales se definen como espacios geográficos cuya cohesión deriva de un tejido social específico, de una base de recursos naturales particular, de unas instituciones y formas de organización propias, y de determinadas formas de producción, intercambio y distribución del ingreso que les dan especificidad regional. En esta concepción el territorio sirve como elemento integrador de agentes, mercados y políticas públicas, al mismo tiempo de ser el objetivo del desarrollo económico. 
- $\mathrm{Al}$ interior de los territorios rurales participan un conjunto de actores (empresas, instituciones, asociaciones, poderes públicos, locales, etc); elementos materiales (empresas, infraestructura, equipamiento) y elementos inmateriales como el saber hacer, las formas seculares de organización y las pautas de comportamiento colectivo. Por tanto, los procesos de desarrollo de los territorios rurales contienen nociones de aprendizaje y el saber hacer y la cultura de organización local constituyen su núcleo fundamental.

- La opinión de la comunidad local se toma como el principal cimiento para construir el proceso de desarrollo. Contar con la gente para desarrollar propuestas sirve para fomentar una cultura de corresponsabilidad y permite que la comunidad de forma colectiva e individual se sienta vinculada a la forma de entender el territorio como un espacio común que provee de bienes, servicios y satisfactores suficientes para vivir con calidad.

- Por ello, la inclusión de la participación como principio se convierte en el proceso básico de democratización de la planificación territorializada del medio rural, que si bien incrementa la complejidad de la gestión del desarrollo, facilita a la larga la organización del territorio y fomenta entre la comunidad local la idea de pertenencia, de cohesión y el sentimiento de formar parte de un proyecto colectivo.

- Finalmente, la gestión pública en beneficio de los territorios rurales debe ser promotora de programas de desarrollo con un carácter de multisectorialidad operativa, fortalecer la visión de comunidad como eje fundamental de integración rural y promover una vida digna y reconstitutiva de las tradiciones y las culturas populares bajo la premisa de que el desarrollo sustentable es posible. 


\section{Bibliografía}

Boisier, Sergio (2004). "Una (re)visión heterodoxa del desarrollo (territorial): un imperativo categórico”. Estudios Sociales: Revista de Investigación Científica, Vol. 12, núm. 23, Hermosillo, México.

Delgadillo, Javier (coordinador) (2008). Enfoque territorial para el desarrollo rural en México. IIEc-UNAM, CRIM-UNAM, El Colegio de Tlaxcala A.C., Unidad de Ciencias del Desarrollo Regional-UAGro, México.

Delgadillo, Javier (2008). "Estrategias territoriales para el desarrollo rural de México”. Revista Ambienta núm. 81. Ministerio de Medio Ambiente y Medio Rural y Marino, Madrid, octubre del 2008.

Echeverri, Rafael (2009). Identidad y territorio en Brasil. Instituto Interamericano de Cooperación para la Agricultura, Secretaría de Desarrollo Territorial del Ministerio de Desarrollo Agrario de Brasil, Sao Paulo.

Guerrero, José Emilio (2008). "Reflexiones sobre una nueva generación de políticas de desarrollo rural”. Revista Ambienta núm. 81. Ministerio de Medio Ambiente y Medio Rural y Marino, Madrid, Octubre del 2008.

Izquierdo, Jaime (2005). Manual para agentes de desarrollo rural. Ministerio de Agricultura, Pesca y Alimentación, Mundi-Prensa, Madrid, 2005 .

Moyano, Eduardo (2008). "Multifuncionalidad, territorio y desarrollo". Revista Ambienta núm. 81. Ministerio de Medio Ambiente y Medio Rural y Marino, Madrid, octubre de 2008.

Schejtman, Alexander y Julio A. Berdegué (2004). Desarrollo territorial rural. Fondo Internacional de Desarrollo Agrícola (FIDA) y Banco Interamericano de Desarrollo (BID), Santiago de Chile.

Torres, Felipe y Javier Delgadillo (2009). Hacia una política territorial del desarrollo rural de México. Convergencia, Revista de Ciencias Sociales, año 16, núm. 50, mayo-agosto, México. 